\title{
NMR and NBO Investigation of Dopamine Properties in Point View of Brain activities
}

\author{
M. MEHDIZADEH BARFORUSHI* \\ Department of Chemistry, College of Basic Sciences, Tehran Science \\ and Research Branch, Islamic Azad University, Tehran, Iran. \\ ${ }^{*}$ Corresponding author E-mail: Maryam.mehdizadeh.b@gmail.com
}

http://dx.doi.org/10.13005/ojc/300443

(Received: September 25, 2014; Accepted: November 10, 2014)

\begin{abstract}
Dopamine dosage is the main reason for panic, fear as well as exhilaration. In this study, it has been shown that the electron negativity of $\mathrm{Cl}, \mathrm{Br}$ and $\mathrm{F}$ ions plays an important role in binding of these ions to dopamine receptors. The nervous quartiles effective drugs have been calculated via NMR and NBO approach. Moreover, the HOMO-LUMO energy gap of dopamine and its halogenated derivatives have been calculated at the B3LYP/6-31G* level. These calculations are so important in order to investigate many other diseases since it can exhibit the ability and relation between electron acceptor represents and electron donation in any brain activities. Finally, the accuracy of calculated result has confirmed by comparing the results to the experimental data.
\end{abstract}

Key words: Dopamine; NMR; NBO;Brain activity.

\section{INTRODUCTION}

Dopamine is a nervous transporter which has a key role in the central nervous system. This nervous transporter has an important role in the normal functions of brain such as learning, memorizing (storage ability in mind), controlling the noise level and adjusting character. Also do-pamine is the brain's Cortex and this Cortex is responsible for human behaviors ${ }^{1-9}$.

Through the decades, dopamine has been always attracted by scientists due to important roles in many diseases such as schizophrenia,
Parkinson's and depression (e.g. increase or decrease dopamine dosage in the body can cause disorder dopamine depression ${ }^{10}$. However the structure and reactivity of this molecule has not been investigated thoroughly ${ }^{11-13}$.

Understanding the structure, reactivity and stability of dopamine derivateis important for understanding the acting mechanism in the body sincethey determine the bonding ability to receptors. In this paper, the effect of electronegativity of atoms in the binding ability to receptors has been investigated in order to improve drug treatment of this common drug.For this purpose, the energy 
levels of dopamine molecules and electronegativity atoms such as chlorine, fluorine, bromine ions are calculated via NMR and NBO method ${ }^{14-17}$.

\section{Computational Methods}

The structures of dopamine and halogenated derivatives were designed primarily using Chem. Bio Draw 12.0 (Scheme 1). The geometry of the systems has been optimized at the B3LYP /6-31G* computational level [18-20]. The optimization, NBO and NMR calculations of all systems are done by density functional theory (DFT) using B3LYP method and the standard 6-31G* basis set, by Gaussian09 suit of programs (Gaussian 09, Revision A.02) in the gas phase $\mathrm{e}^{21-24}$.

Mullikan charges on the atoms, dipole moments, and geometry parameters such as bond lengths were also determined by the same method. The energy barrier for rotation around the tow bond in the dopamine moleculeis also assessed and the results are represented in Table 1 and Fig. ${ }^{25-27}$.

\section{RESULTS AND DISCUSSION}

\section{Energies}

The $E_{\text {Hомо }}, E_{\text {Lumo }}$ and HOMO-LUMO energygap $(\mathrm{Eg} ; \Delta)$ of dopamine and halogenated derivatives were calculated using the B3LYP method and $6-31 G^{*}$ basis set and the results are presented in Table 4-9 and Table S3-S8
(Supplementary materials) ${ }^{28}$.

The energy levels are so important since the energy level for LUMO as an electron acceptor represents the ability to obtain an electron and the energy level of HOMOrepresentsthe ability to donate an electron. As shown in Table 4-9, the $(\mathrm{Cl})_{2}$-Dop moleculeh as the lowest energy gap is -175.7456 $\mathrm{Kcal} / \mathrm{mol}$ and the dopamine molecule has the largest energy gap is-130.9417 Kcal/mol ${ }^{29-31}$.

\section{Geometries}

The structures of dopamine and halogenated derivatives were designed primarily using of Chem. Bio Draw 12.0 in scheme 1 and the obtained results were compared to other derivate at the end. The optimization, NBO and NMR calculations of the whole systems are done by density functional theory (DFT) using B3LYP method and the standard 6-31G basis set using Gaussian 09. The optimized geometrical parameters, such as Dipole moment (Debye), energy of structure formation (HF;kcal/mol) and enthalpies $(\Delta \mathrm{H})$, Gibbs free energy $(\Delta \mathrm{G})$ are listed in Table 4-9.As shown in Table 4-9, the $(\mathrm{Br}) 2$ - Dop moleculehasthe lowest $\Delta G$ is $72.059013 \mathrm{Kcal} / \mathrm{mol}$ and the dopamine molecule has the largest $\Delta \mathrm{G}$ is $91.416315 \mathrm{Kcal} / \mathrm{mol}$. The molecule has the lowest $\Delta \mathrm{H}$ is $102.29162 \mathrm{Kcal} /$ mol and the dopamine molecule has the largest $\Delta \mathrm{H}$ is $122.32726 \mathrm{Kcal} / \mathrm{mol}$.

Table 1: The calculated energy barrier of rotation around bonds $C_{1}-O_{10}$ and $C_{2}-O_{11}$

\begin{tabular}{|c|c|c|c|}
\hline \multicolumn{2}{|c|}{ Rout $1\left(\mathrm{C}_{6}-\mathrm{C}_{1}-\mathrm{O}_{10}-\mathrm{H}_{21}\right)$} & \multicolumn{2}{|c|}{ Rout $2\left(\mathrm{C}_{3}-\mathrm{C}_{2}-\mathrm{O}_{11}-\mathrm{H}_{22}\right)$} \\
\hline Angstroms and Degrees & $\mathrm{E}(\mathrm{k}(\mathrm{cal} / \mathrm{mol})$ & Angstroms and Degrees & $\mathrm{E}(\mathrm{k}(\mathrm{cal} / \mathrm{mol})$ \\
\hline-15.2 & -2.32175 & -166.77 & -8.09475 \\
\hline-0.202 & -1.8825 & 13.22 & -2.259 \\
\hline 14.8 & -2.32175 & 28.22 & -1.69425 \\
\hline 29.8 & -1.69425 & 43.22 & -0.94125 \\
\hline 44.8 & -0.94125 & 58.22 & -0.251 \\
\hline 59.8 & -0.251 & 73.22 & 0 \\
\hline 74.8 & 0 & 88.22 & -0.31375 \\
\hline 89.8 & -0.43925 & 103.22 & -1.19225 \\
\hline 104.8 & -1.44325 & 118.22 & -1.8825 \\
\hline 119.8 & -3.07475 & 133.22 & -4.518 \\
\hline 134.8 & -4.95725 & 148.22 & -6.4005 \\
\hline 149.8 & -6.83975 & 163.22 & -7.781 \\
\hline 164.8 & -8.22025 & 178.22 & -8.4085 \\
\hline
\end{tabular}


Table 2: Computed chemical shifts for selected atoms

\begin{tabular}{|c|c|c|c|c|c|c|}
\hline Structure & Atom number & $\sigma 11$ & $\sigma 22$ & $\sigma 33$ & бiso & $\Delta \sigma$ \\
\hline$(\mathrm{OH}) 2-\mathrm{DOP} *$ & C1 & -6.7203 & 35.6456 & 129.586 & 52.8371 & 115.1234 \\
\hline (F)2-DOP* & & -17.4526 & 17.9589 & 78.9894 & 26.4985 & 78.7362 \\
\hline $\mathrm{F}(\mathrm{OH})-\mathrm{DOP}{ }^{*}$ & & -16.694 & 22.9613 & 76.7965 & 27.68793333 & 73.66285 \\
\hline (Cl)2-DOP* & & -22.6423 & 57.3594 & 114.0318 & 49.58297 & 96.67325 \\
\hline $\mathrm{Cl}(\mathrm{OH})-\mathrm{DOP} *$ & & 3.7164 & 62.4586 & 123.6434 & 63.2728 & 90.5559 \\
\hline$(\mathrm{Br}) 2-\mathrm{DOP} *$ & & -25.6558 & 56.5618 & 110.3614 & 47.08913333 & 94.9084 \\
\hline $\mathrm{Br}(\mathrm{OH})-\mathrm{DOP}{ }^{*}$ & & 11.7828 & 69.3353 & 126.8483 & 69.32213333 & 86.28925 \\
\hline$(\mathrm{OH}) 2-\mathrm{DOP}^{*}$ & $\mathrm{C} 2$ & -4.2227 & 35.8531 & 130.5894 & 54.07326667 & 114.7742 \\
\hline (F)2-DOP* & & -12.7193 & 18.0642 & 79.269 & 28.2 & 76.59 \\
\hline $\mathrm{F}(\mathrm{OH})-\mathrm{DOP}{ }^{*}$ & & -8.3722 & 24.9618 & 21.9099 & 12.83316667 & 13.6151 \\
\hline (Cl)2-DOP* & & -17.9397 & 59.6516 & 114.7442 & 52.15203 & 93.88825 \\
\hline $\mathrm{Cl}(\mathrm{OH})-\mathrm{DOP}^{*}$ & & 8.0263 & 26.0354 & 122.4972 & 52.1863 & 105.4664 \\
\hline (Br)2-DOP* & & -20.9239 & 59.6319 & 111.1917 & 49.96656667 & 91.8377 \\
\hline $\mathrm{Br}(\mathrm{OH})-\mathrm{DOP}{ }^{*}$ & & 29.0368 & 34.451 & 131.1433 & 64.87703 & 99.3994 \\
\hline$(\mathrm{OH}) 2-\mathrm{DOP}{ }^{*}$ & C8 & 19.9281 & 32.2393 & 170.3295 & 74.16563333 & 144.2458 \\
\hline$(F) 2-D O P^{*}$ & & 129.9135 & 36.9611 & 64.7332 & 143.86 & 31.29 \\
\hline $\mathrm{F}(\mathrm{OH})-\mathrm{DOP}{ }^{*}$ & & 130.0042 & 36.7783 & 64.3277 & 77.03673333 & -19.06355 \\
\hline$(\mathrm{Cl}) 2-\mathrm{DOP}^{*}$ & & 129.945 & 36.9932 & 164.9629 & 110.6337 & 81.4938 \\
\hline $\mathrm{Cl}(\mathrm{OH})-\mathrm{DOP}{ }^{*}$ & & 9.7885 & 36.8712 & 164.4894 & 70.38303 & 141.1596 \\
\hline (Br)2-DOP* & & 129.7864 & 36.9806 & 165.0564 & 110.6078 & 81.6729 \\
\hline $\mathrm{Br}(\mathrm{OH})-\mathrm{DOP}{ }^{*}$ & & 20.2364 & 31.8799 & 170.9779 & 74.36473333 & 144.91975 \\
\hline
\end{tabular}

The isotropic chemical shielding $\sigma_{\text {iso }}$ parameters are average of parameters, $\sigma_{11}, \sigma_{22}$ and $\sigma_{33}>33>22>11$ *Dop=dopamine

Table 3: Computed chemical shifts for selected atoms

\begin{tabular}{|c|c|c|c|c|c|c|}
\hline Structure & Atom number & $\sigma 11$ & $\sigma 22$ & $\sigma 33$ & oiso & $\Delta \sigma$ \\
\hline$\overline{(\mathrm{OH}) 2-\mathrm{DOP} *}$ & $\mathrm{~N}$ & 95.9945 & 29.4999 & 242.7177 & 122.7373667 & 179.9705 \\
\hline (F)2-DOP* & & 191.716 & 25.3634 & 41.97 & 219.68 & 33.43 \\
\hline $\mathrm{F}(\mathrm{OH})-\mathrm{DOP}{ }^{*}$ & & 190.9571 & 25.0724 & 41.8411 & 85.95686667 & -66.17365 \\
\hline (Cl)2-DOP* & & 191.5971 & 25.4218 & 241.9345 & 152.9845 & 133.4251 \\
\hline $\mathrm{Cl}(\mathrm{OH})-\mathrm{DOP}{ }^{*}$ & & 0.6262 & 25.0737 & 241.9851 & 89.22833333 & 229.13515 \\
\hline$(\mathrm{Br}) 2-\mathrm{DOP}^{*}$ & & 191.5634 & 25.4276 & 241.9487 & 152.9799 & 133.4532 \\
\hline $\mathrm{Br}(\mathrm{OH})-\mathrm{DOP}^{*}$ & & 96.2871 & 29.4256 & 243.2144 & 122.9757 & 180.3581 \\
\hline$(\mathrm{OH}) 2-\mathrm{DOP}^{*}$ & $\mathrm{H} 19$ & 24.7994 & 28.992 & 41.3526 & 31.71467 & 14.4569 \\
\hline (F)2-DOP* & & 23.7725 & 29.4723 & 40.0824 & 31.1 & 13.46 \\
\hline $\mathrm{F}(\mathrm{OH})-\mathrm{DOP}{ }^{*}$ & & 23.8236 & 29.5709 & 40.1099 & 31.16813333 & 13.41265 \\
\hline (Cl)2-DOP* & & 23.7761 & 29.5058 & 40.1742 & 31.15203333 & 13.53325 \\
\hline $\mathrm{Cl}(\mathrm{OH})-\mathrm{DOP} *$ & & 3.8093 & 29.5853 & 40.1726 & 24.5224 & 23.4753 \\
\hline$(\mathrm{Br}) 2-\mathrm{DOP}{ }^{*}$ & & 23.7379 & 29.4884 & 40.2316 & 31.15263 & 13.61845 \\
\hline $\mathrm{Br}(\mathrm{OH})-\mathrm{DOP}^{*}$ & & 24.719 & 28.9215 & 41.4097 & 31.6834 & 14.58945 \\
\hline$(\mathrm{OH}) 2-\mathrm{DOP}{ }^{*}$ & $\mathrm{H} 20$ & 25.6399 & 31.2533 & 39.7743 & 32.2225 & 11.3277 \\
\hline$(F) 2-D O P^{*}$ & & 25.2384 & 31.3865 & 38.977 & 31.86 & 10.66 \\
\hline $\mathrm{F}(\mathrm{OH})-\mathrm{DOP}{ }^{*}$ & & 25.2569 & 31.4527 & 38.9689 & 31.89283333 & 10.6141 \\
\hline (Cl)2-DOP* & & 25.2443 & 31.4942 & 38.9987 & 31.9124 & 10.62945 \\
\hline $\mathrm{Cl}(\mathrm{OH})-\mathrm{DOP}{ }^{*}$ & & 5.2442 & 31.5347 & 38.9681 & 25.249 & 20.57865 \\
\hline$(\mathrm{Br}) 2-\mathrm{DOP}{ }^{*}$ & & 25.2012 & 31.5541 & 38.9776 & 31.91097 & 10.59995 \\
\hline $\mathrm{Br}(\mathrm{OH})-\mathrm{DOP}^{*}$ & & 25.4854 & 31.2492 & 39.7694 & 32.168 & 11.4021 \\
\hline
\end{tabular}

The isotropic chemical shielding $\sigma_{\text {iso }}$ parameters are average of parameters, $\sigma_{11}, \sigma_{22}$ and $\sigma_{33}>33>22>11$ *Dop=dopamine 


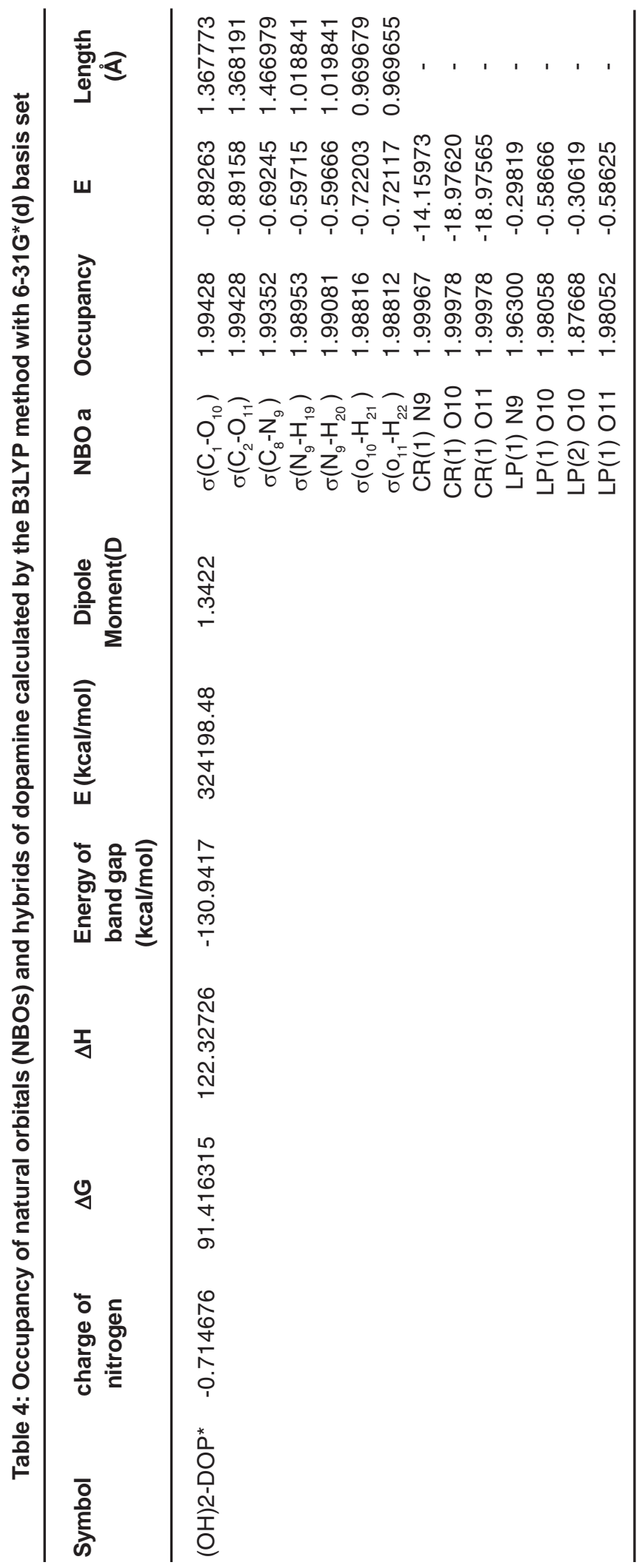




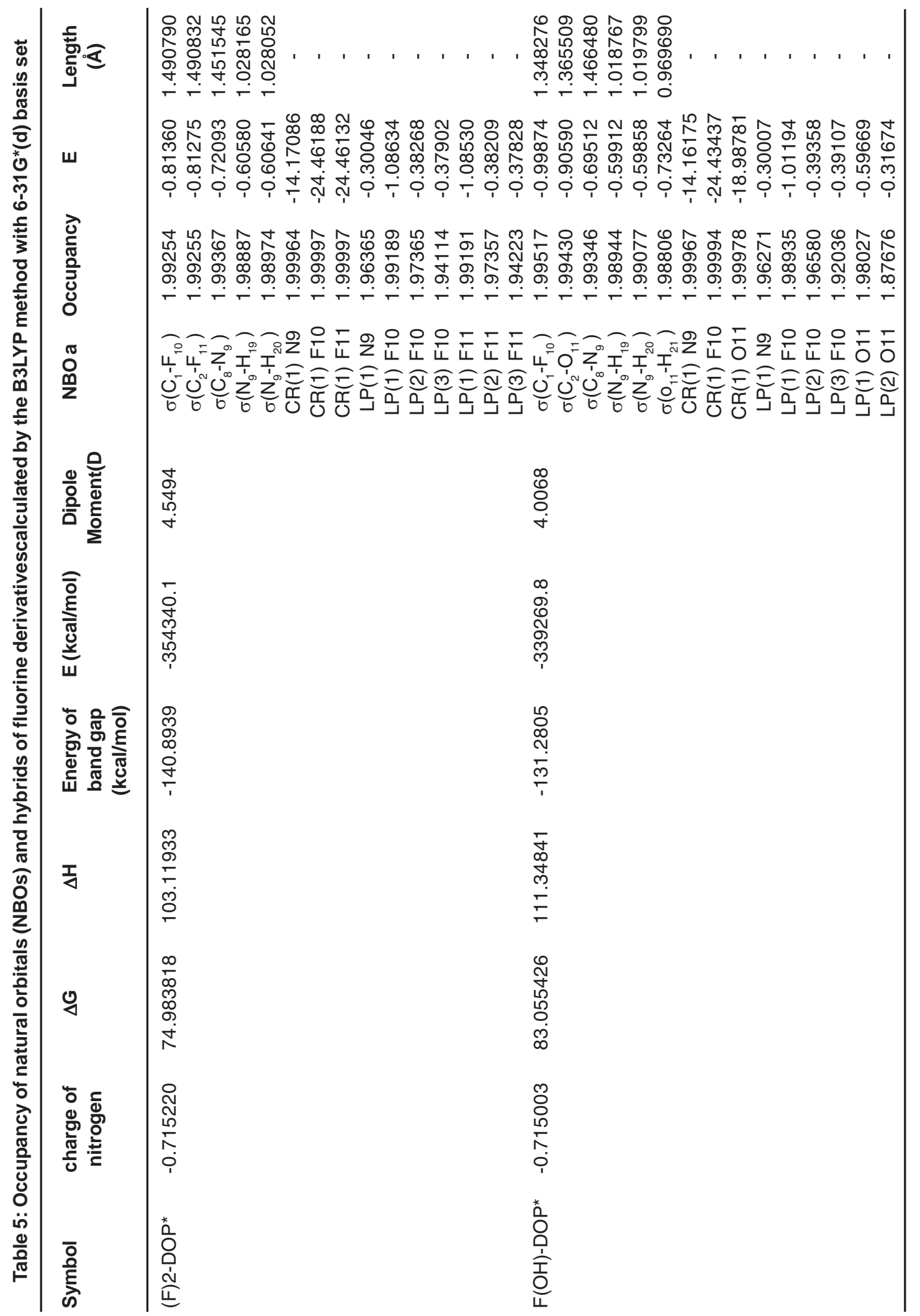




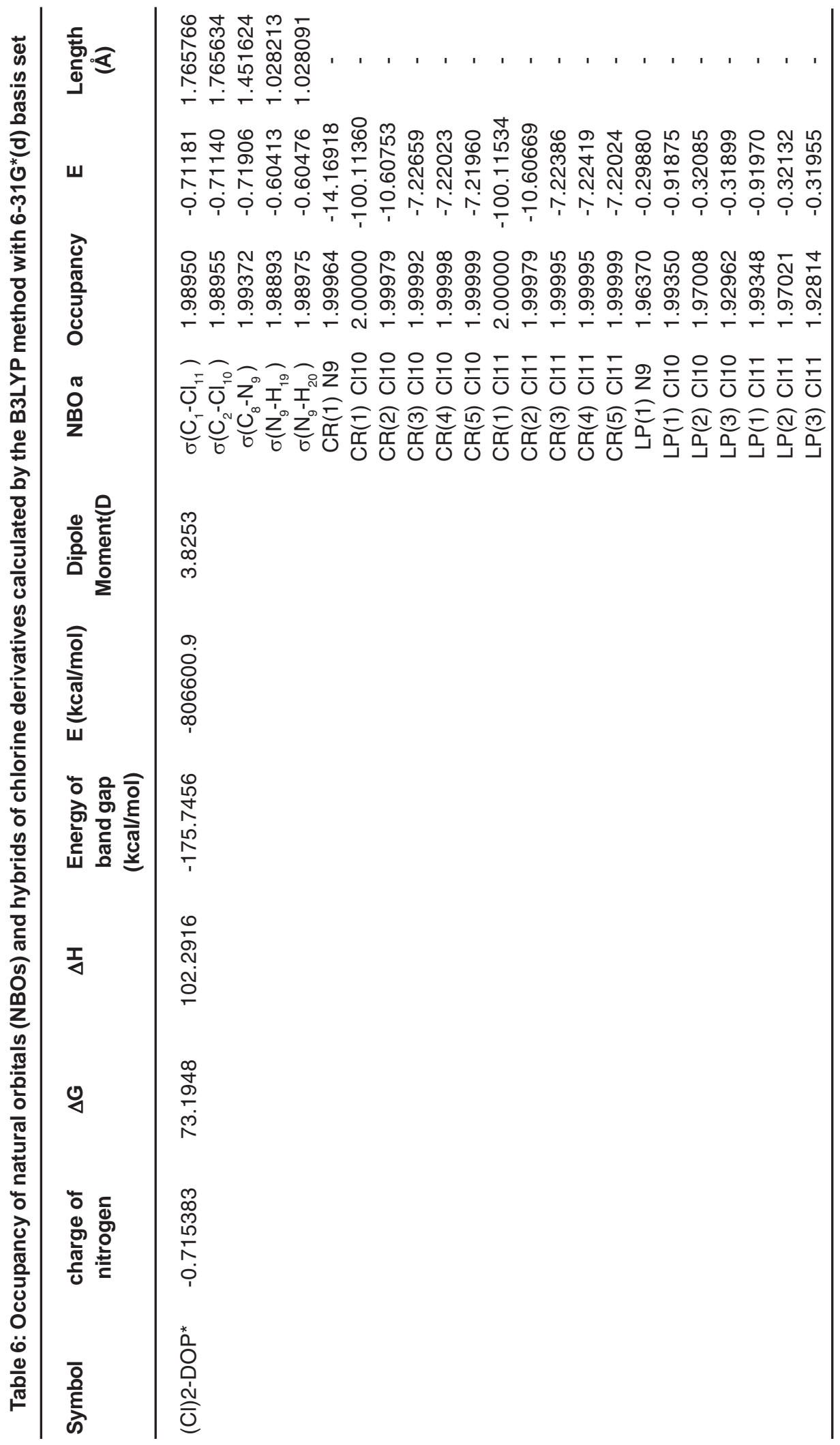




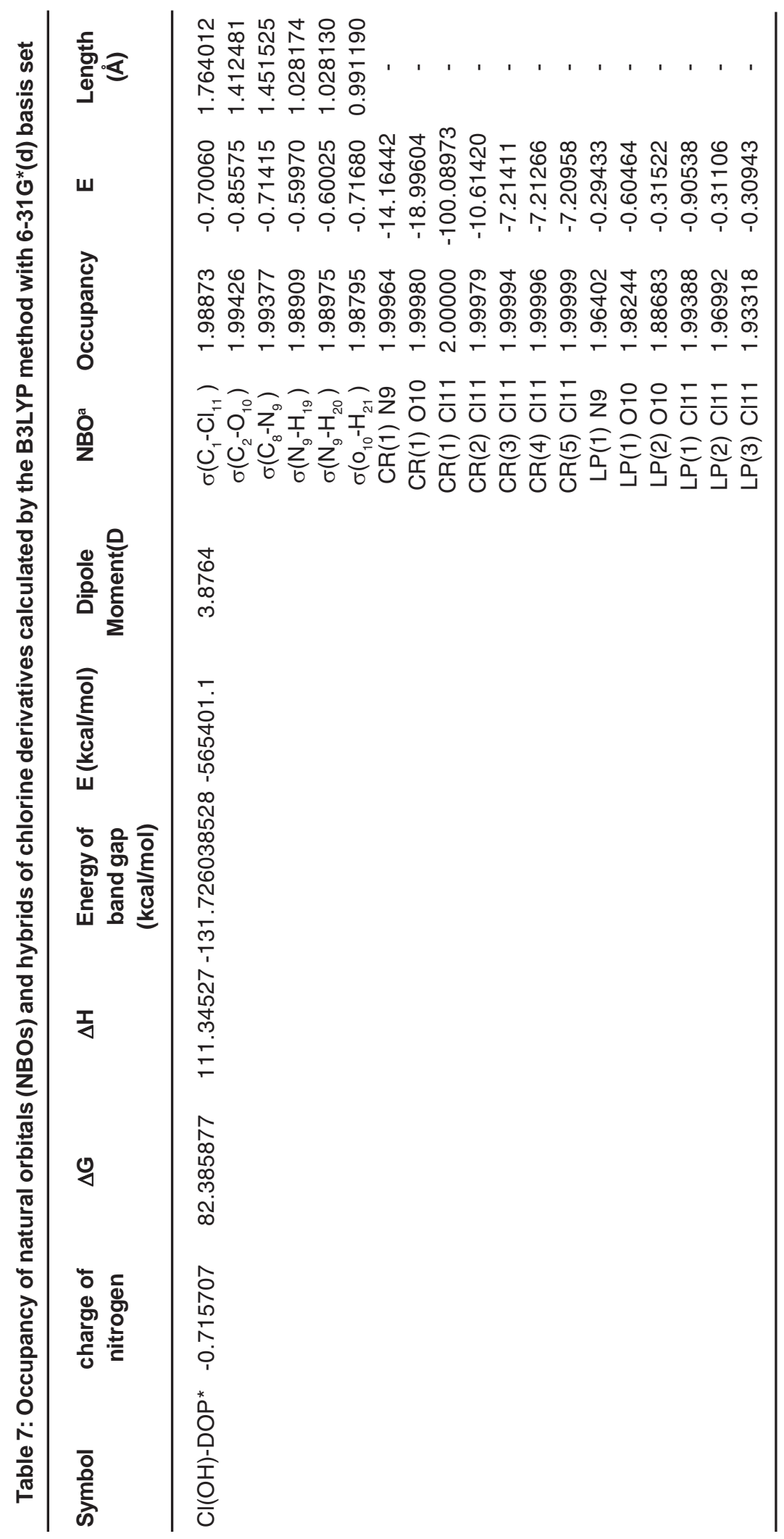




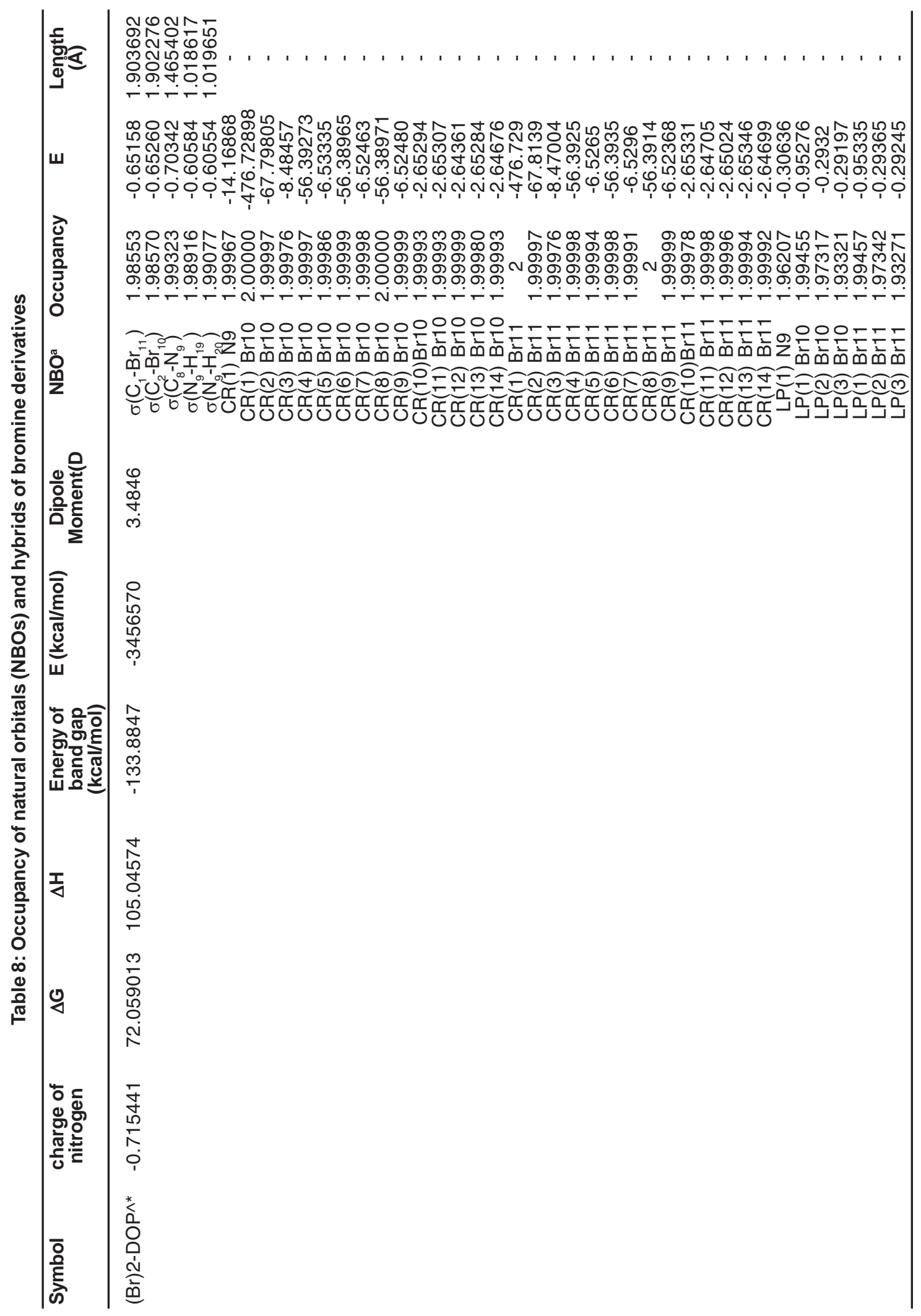




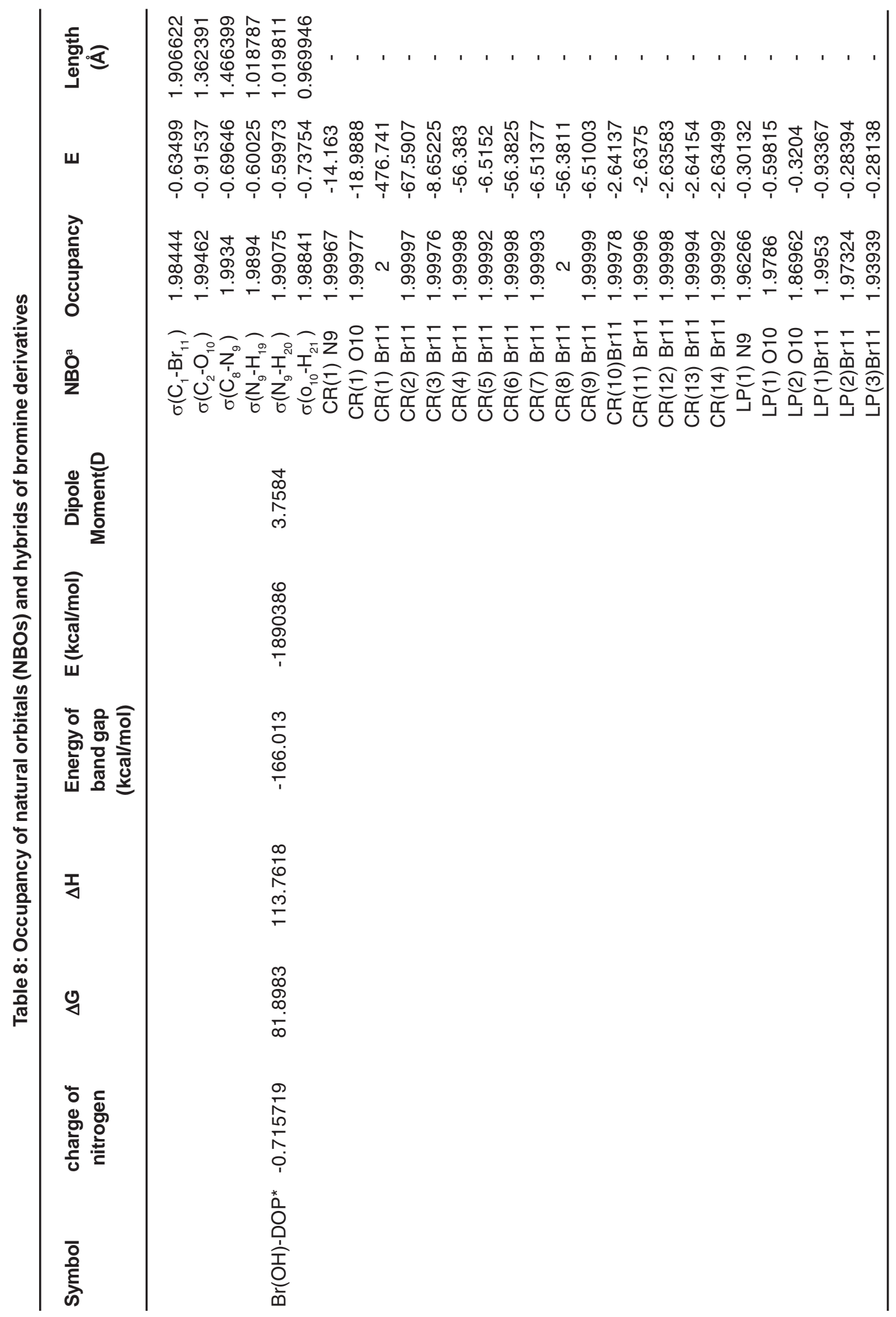




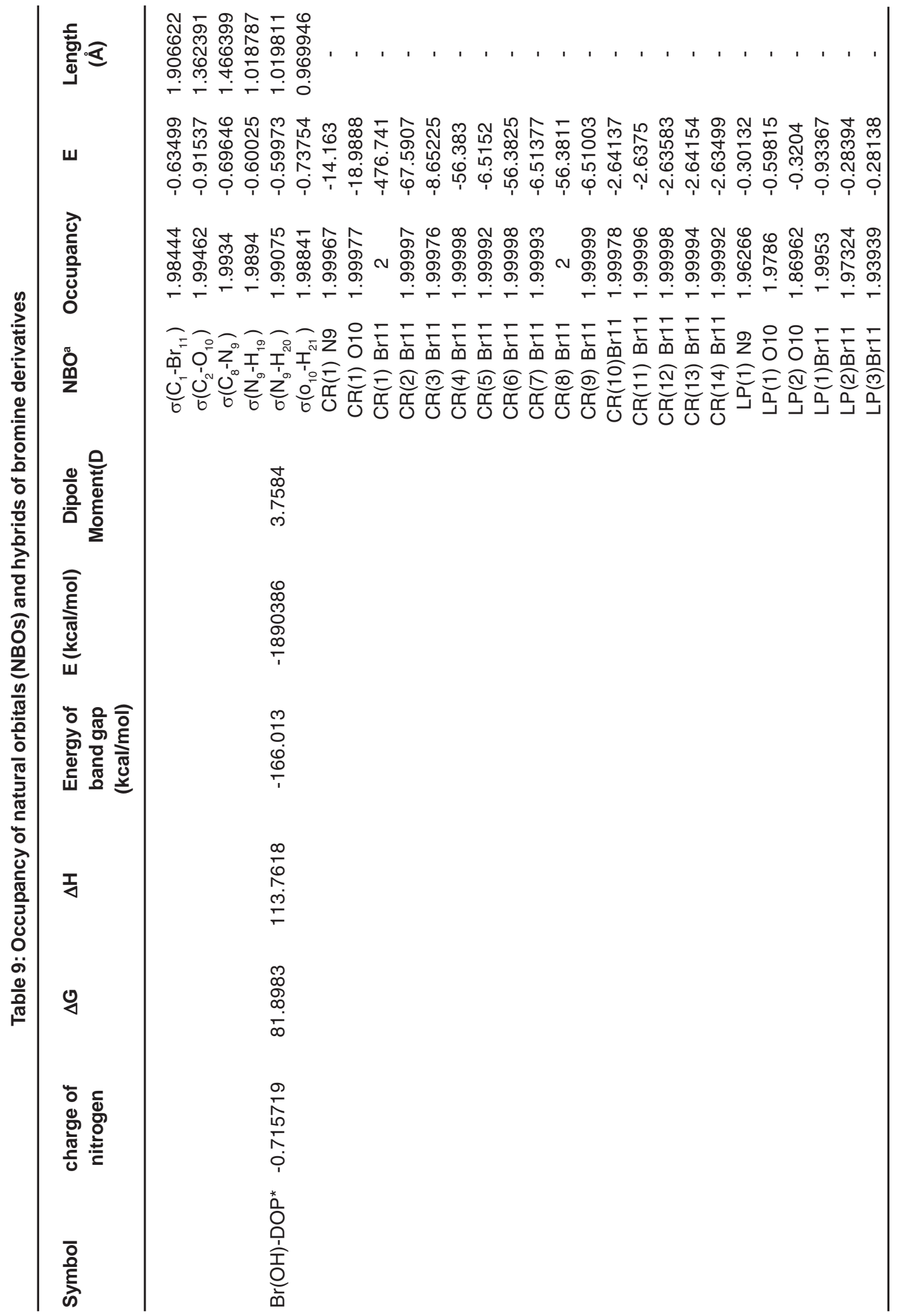


Table S1: Computed chemical shifts for selected atoms

\begin{tabular}{|c|c|c|c|c|}
\hline Structure & Atom number & $\eta$ & $\kappa$ & Charge \\
\hline$(\mathrm{OH}) 2-\mathrm{DOP}^{*}$ & $\mathrm{C} 1$ & 0.552007 & 0.378372093 & 0.327738 \\
\hline (F)2-DOP* & & 0.674621696 & 0.48806174 & 0.581972 \\
\hline $\mathrm{F}(\mathrm{OH})-\mathrm{DOP}{ }^{*}$ & & 0.807502696 & 0.151672095 & 0.310302 \\
\hline (Cl)2-DOP* & & 1.241321 & -0.17069 & -0.07644 \\
\hline $\mathrm{Cl}(\mathrm{OH})-\mathrm{DOP} *$ & & -1.6284 & 0.02036739 & -0.111033 \\
\hline$(\mathrm{Br}) 2-\mathrm{DOP}^{\star}$ & & 1.299425551 & -0.208929459 & 0.048784 \\
\hline $\mathrm{Br}(\mathrm{OH})-\mathrm{DOP}^{*}$ & & 1.000457763 & -0.000343283 & 0.047116 \\
\hline$(\mathrm{OH}) 2-\mathrm{DOP}^{*}$ & $\mathrm{C} 2$ & 0.52375621 & 0.405457 & 0.315797 \\
\hline (F)2-DOP* & & 0.60278251 & 0.595417964 & 0.003529 \\
\hline $\mathrm{F}(\mathrm{OH})-\mathrm{DOP}{ }^{*}$ & & 3.6724666 & -1.201564621 & 0.305575 \\
\hline (Cl)2-DOP* & & 1.239633 & -0.16957 & -0.07595 \\
\hline $\mathrm{Cl}(\mathrm{OH})-\mathrm{DOP}^{*}$ & & -52.3018 & 0.685351 & 0.362558 \\
\hline (Br)2-DOP* & & 1.315730904 & -0.21947446 & 0.048189 \\
\hline $\mathrm{Br}(\mathrm{OH})-\mathrm{DOP}{ }^{*}$ & & 0.081704 & 0.89395 & 0.328631 \\
\hline$(\mathrm{OH}) 2-\mathrm{DOP}$ * & $\mathrm{C8}$ & 0.128023138 & 0.836289 & -0.128391 \\
\hline (F)2-DOP* & & -13.8256 & -4.052946663 & -0.340644 \\
\hline $\mathrm{F}(\mathrm{OH})-\mathrm{DOP}^{*}$ & & 7.335404476 & -1.838942392 & -0.133027 \\
\hline (Cl)2-DOP* & & -1.710899479 & 6.308816348 & -0.134595 \\
\hline $\mathrm{Cl}(\mathrm{OH})-\mathrm{DOP}{ }^{*}$ & & -67.0237 & 0.64987 & -0.13307 \\
\hline$(\mathrm{Br}) 2-\mathrm{DOP} *$ & & -1.70447 & 6.262591 & -0.13434 \\
\hline $\mathrm{Br}(\mathrm{OH})-\mathrm{DOP} *$ & & 0.1205167 & 0.845516994 & -0.129605 \\
\hline
\end{tabular}

Table S2: Computed chemical shifts for selected atoms

\begin{tabular}{|c|c|c|c|c|}
\hline Structure & Atom number & $\eta$ & $\kappa$ & Charge \\
\hline$(\mathrm{OH}) 2-\mathrm{DOP} *$ & $\mathrm{~N}$ & -0.554212496 & 1.906395171 & -0.714647 \\
\hline (F)2-DOP* & & 0.936090259 & -3.280343256 & -0.407913 \\
\hline $\mathrm{F}(\mathrm{OH})-\mathrm{DOP}{ }^{*}$ & & 3.760213469 & -1.224908125 & -0.715729 \\
\hline (Cl)2-DOP* & & -1.86819 & 7.602459 & -0.7154 \\
\hline $\mathrm{Cl}(\mathrm{OH})-\mathrm{DOP}^{*}$ & & -128.3092667 & 0.79741787 & -0.715707 \\
\hline$(\mathrm{Br}) 2-\mathrm{DOP} *$ & & -1.86735 & 7.594614 & -0.71544 \\
\hline $\mathrm{Br}(\mathrm{OH})-\mathrm{DOP}{ }^{*}$ & & -0.55607 & 1.91013 & -0.71502 \\
\hline$(\mathrm{OH}) 2-\mathrm{DOP} *$ & $\mathrm{H} 19$ & 0.43501 & 0.493439335 & 0.296342 \\
\hline (F)2-DOP* & & 0.634552 & 0.543629765 & 0.310717 \\
\hline $\mathrm{F}(\mathrm{OH})-\mathrm{DOP}{ }^{*}$ & & 0.642747705 & 0.294216612 & 0.298807 \\
\hline (Cl)2-DOP* & & 0.635069181 & 0.301175136 & 0.300622 \\
\hline $\mathrm{Cl}(\mathrm{OH})-\mathrm{DOP}^{*}$ & & 10.1258 & -0.417693114 & 0.298418 \\
\hline$(\mathrm{Br}) 2-\mathrm{DOP}{ }^{*}$ & & 0.633387 & 0.302703 & 0.300477 \\
\hline $\mathrm{Br}(\mathrm{OH})-\mathrm{DOP}{ }^{*}$ & & 0.432076 & 0.496426 & 0.297998 \\
\hline$(\mathrm{OH}) 2-\mathrm{DOP}{ }^{*}$ & $\mathrm{H} 20$ & 0.743319474 & 0.20571089 & 0.290714 \\
\hline (F)2-DOP* & & 0.863861 & 0.199592525 & 0.2761 \\
\hline $\mathrm{F}(\mathrm{OH})-\mathrm{DOP}{ }^{*}$ & & 0.875599438 & 0.096295216 & 0.293655 \\
\hline (Cl)2-DOP* & & 0.881969434 & 0.091214448 & 0.294312 \\
\hline $\mathrm{Cl}(\mathrm{OH})-\mathrm{DOP}^{*}$ & & 12.5714 & -0.55916 & 0.293424 \\
\hline$(\mathrm{Br}) 2-\mathrm{DOP}{ }^{*}$ & & 0.899 & 0.077713 & 0.294229 \\
\hline $\mathrm{Br}(\mathrm{OH})-\mathrm{DOP}^{*}$ & & 0.758255 & 0.192971 & 0.293085 \\
\hline
\end{tabular}


Table S3: Occupancy of natural orbitals (NBOs) and hybrids of dopamine calculated by the B3LYP method with 6-31G*(d) basis set

\begin{tabular}{llll}
\hline Symbol & NBO a & Occupancy & Hybrid \\
\hline$(\mathrm{OH}) 2-\mathrm{DOP}{ }^{*}$ & $\sigma\left(\mathrm{C}_{1}-\mathrm{O}_{10}\right)$ & 1.99428 & $0.5771\left(\mathrm{sp}^{3}\right)_{\mathrm{C} 1}+0.8166\left(\mathrm{sp}^{1.93}\right)_{\mathrm{O} 10}$ \\
& $\sigma\left(\mathrm{C}_{2}-\mathrm{O}_{11}\right)$ & 1.99428 & $0.5774\left(\mathrm{sp}^{2.98}\right)_{\mathrm{C} 2}+0.8165\left(\mathrm{sp}^{1.94}\right)_{\mathrm{O} 11}$ \\
& $\sigma\left(\mathrm{C}_{8}-\mathrm{N}_{9}\right)$ & 1.99352 & $0.6368\left(\mathrm{sp}^{3.17}\right)_{\mathrm{C} 8}+0.7710\left(\mathrm{sp}^{2.19}\right)_{\mathrm{N} 9}$ \\
& $\sigma\left(\mathrm{N}_{9}-\mathrm{H}_{19}\right)$ & 1.98953 & $0.8299\left(\mathrm{sp}^{3.23}\right)_{\mathrm{Ng}}+0.5579(\mathrm{~s})_{\mathrm{H} 19}$ \\
& $\sigma\left(\mathrm{N}_{9}-\mathrm{H}_{20}\right)$ & 1.99081 & $0.8282\left(\mathrm{sp}^{3.28}\right)_{\mathrm{N} 9}+0.5605(\mathrm{~s})_{\mathrm{H} 20}$ \\
& $\sigma\left(\mathrm{O}_{10}-\mathrm{H}_{21}\right)$ & 1.98816 & $0.8625\left(\mathrm{sp}^{3.92}\right)_{\mathrm{O} 10}+0.5060(\mathrm{~s})_{\mathrm{H} 21}$ \\
& $\sigma\left(\mathrm{O}_{11}-\mathrm{H}_{22}\right)$ & 1.98812 & $0.8626\left(\mathrm{sp}^{3.92}\right)_{\mathrm{O} 11}+0.5059(\mathrm{~s})_{\mathrm{H} 22}$ \\
& $\mathrm{CR}(1) \mathrm{N9}$ & 1.99967 & $\mathrm{~s}$ \\
$\mathrm{CR}(1) \mathrm{O} 10$ & 1.99978 & $\mathrm{~s}$ \\
$\mathrm{CR}(1) \mathrm{O} 11$ & 1.99978 & $\mathrm{~s}$ \\
& $\mathrm{LP}(1) \mathrm{N9}$ & 1.96300 & $\mathrm{sp}^{3.62}$ \\
& $\mathrm{LP}(1) \mathrm{O} 10$ & 1.98058 & $\mathrm{sp}^{1.19}$ \\
& $\mathrm{LP}(2) \mathrm{O} 10$ & 1.87668 & $\mathrm{p}$ \\
$\mathrm{LP}(1) \mathrm{O} 11$ & 1.98052 & $\mathrm{sp}^{1.19}$ \\
\hline
\end{tabular}

Table S4: Occupancy of natural orbitals (NBOs) and hybrids of fluorine derivativescalculated by the B3LYP method with 6-31G*(d) basis set

\begin{tabular}{|c|c|c|c|}
\hline Symbol & NBO a & Occupancy & Hybrid \\
\hline \multirow[t]{15}{*}{$(\mathrm{F}) 2-\mathrm{DOP}^{*}$} & $\sigma\left(C_{1}-F_{10}\right)$ & 1.99254 & $0.5385\left(\mathrm{sp}^{3.64}\right)_{\mathrm{c} 1}+0.8426\left(\mathrm{sp}^{3.98}\right)_{\mathrm{E} 10}$ \\
\hline & $\sigma\left(\mathrm{C}_{2}-\mathrm{F}_{11}^{10}\right)$ & 1.99255 & $0.5388\left(\mathrm{sp}^{3.62}\right)+0.8424\left(\mathrm{sp}^{3.98}\right)$ \\
\hline & $\sigma\left(\mathrm{C}_{8}^{2}-\mathrm{N}_{9}\right)^{11 \prime}$ & 1.99367 & $0.6402\left(\mathrm{sp}^{3.12}\right)_{\mathrm{c} 8}^{\mathrm{C2}}+0.7682\left(\mathrm{sp}^{2.08}\right)^{11}$ \\
\hline & $\sigma\left(\mathrm{N}_{9}^{8}-\mathrm{H}_{19}\right)$ & 1.98887 & $0.8317\left(\mathrm{sp}^{3.14}\right)_{\mathrm{N} 9}^{\mathrm{co}}+0.5553^{*}(\mathrm{~s})_{\mathrm{H} 19}$ \\
\hline & $\sigma\left(\mathrm{N}_{9}^{9}-\mathrm{H}_{20}\right)$ & 1.98974 & $0.8298\left(\mathrm{sp}^{3.17}\right)_{\mathrm{N} 9}+0.5581(\mathrm{~s})_{\mathrm{H} 2 \mathrm{O}}$ \\
\hline & $\mathrm{CR}(1) \mathrm{N} 9$ & 1.99964 & $\mathrm{p}$ \\
\hline & $\mathrm{CR}(1) \mathrm{F} 10$ & 1.99997 & $s$ \\
\hline & $\mathrm{CR}(1) \mathrm{F} 11$ & 1.99997 & s \\
\hline & LP(1) N9 & 1.96365 & $\mathrm{sp}^{4.15}$ \\
\hline & $\mathrm{LP}(1) \mathrm{F} 10$ & 1.99189 & $\mathrm{sp}^{0.26}$ \\
\hline & LP(2) F10 & 1.97365 & $\mathrm{p}$ \\
\hline & LP(3) F10 & 1.94114 & $\mathrm{p}$ \\
\hline & $\mathrm{LP}(1) \mathrm{F} 11$ & 1.99191 & $\mathrm{sp}^{0.26}$ \\
\hline & LP(2) F11 & 1.97357 & $\mathrm{p}$ \\
\hline & LP(3) F11 & 1.94223 & $\mathrm{p}$ \\
\hline \multirow[t]{15}{*}{$\mathrm{F}(\mathrm{OH})-\mathrm{DOP} *$} & $\sigma\left(\mathrm{C}_{1}-\mathrm{F}_{10}\right)$ & 1.99517 & $0.5260\left(\mathrm{sp}^{3.45} \mathrm{~d}^{0.01}\right)_{\mathrm{C} 1}+0.8505\left(\mathrm{sp}^{2.30}\right)_{\mathrm{F} 10}$ \\
\hline & $\sigma\left(\mathrm{C}_{2}-\mathrm{O}_{11}\right)$ & 1.99430 & $0.5786\left(\mathrm{sp}^{2.93} \mathrm{~d}^{0.01}\right)_{\mathrm{c} 2}+0.8156\left(\mathrm{sp}^{1.94}\right)_{011}$ \\
\hline & $\sigma\left(\mathrm{C}_{8}^{2}-\mathrm{N}_{9}\right)$ & 1.99346 & $0.6373\left(\mathrm{sp}^{3.16} \mathrm{~d}^{0.01}\right)_{\mathrm{co}}+0.7706\left(\mathrm{sp}^{2.19}\right)$ \\
\hline & $\sigma\left(\mathrm{N}_{9}-\mathrm{H}_{19}\right)$ & 1.98944 & $0.8301\left(\mathrm{sp}^{3.23}\right)_{\mathrm{Ng}}+0.5576(\mathrm{~s})_{\mathrm{H} 19}$ \\
\hline & $\sigma\left(\mathrm{N}_{9}^{9}-\mathrm{H}_{20}\right)$ & 1.99077 & $0.8285\left(\mathrm{sp}^{3.27}\right)_{\mathrm{N9}}+0.5600(\mathrm{~s})_{\mathrm{H} 2 \mathrm{O}}$ \\
\hline & $\sigma\left(O_{11}-H_{21}\right)$ & 1.98806 & $0.8635\left(\mathrm{sp}^{3.89} \mathrm{~d}^{0.01}\right) \mathrm{O} 11+0.5043(\mathrm{~s})_{\mathrm{H} 21}$ \\
\hline & $\mathrm{CR}(1) \mathrm{N} 9$ & 1.99967 & $\mathrm{~s}$ \\
\hline & $\mathrm{CR}(1) \mathrm{F} 10$ & 1.99994 & $\mathrm{~s}$ \\
\hline & $\mathrm{CR}(1) 011$ & 1.99978 & $\mathrm{~s}$ \\
\hline & LP(1) N9 & 1.96271 & $\mathrm{sp}^{3.63}$ \\
\hline & $\mathrm{LP}(1) \mathrm{F} 10$ & 1.98935 & $\mathrm{sp}^{0.44}$ \\
\hline & LP(2) F10 & 1.96580 & $\mathrm{sp}^{99.99} \mathrm{~d}^{0.31}$ \\
\hline & LP(3) F10 & 1.92036 & $\mathrm{p}$ \\
\hline & LP(1) 011 & 1.98027 & $\mathrm{sp}^{1.19}$ \\
\hline & LP(2) 011 & 1.87676 & $\mathrm{p}$ \\
\hline
\end{tabular}


Table S5: Occupancy of natural orbitals (NBOs) and hybrids of chlorine derivatives calculated by the B3LYP method with $6-31 G^{*}(d)$ basis set

\begin{tabular}{|c|c|c|c|}
\hline Symbol & $\mathrm{NBO}^{\mathrm{a}}$ & Occupancy & Hybrid \\
\hline$(\mathrm{Cl}) 2-\mathrm{DOP} *$ & $\begin{array}{l}\sigma\left(\mathrm{C}_{1}-\mathrm{Cl}_{11}\right) \\
\sigma\left(\mathrm{C}_{2}-\mathrm{Cl}_{10}\right) \\
\sigma\left(\mathrm{C}_{8}-\mathrm{N}_{9}\right) \\
\sigma\left(\mathrm{N}_{9}-\mathrm{H}_{19}\right) \\
\sigma\left(\mathrm{N}_{9}-\mathrm{H}_{20}\right) \\
\mathrm{CR}(1) \mathrm{N} 9 \\
\mathrm{CR}(1) \mathrm{Cl} 10 \\
\mathrm{CR}(2) \mathrm{Cl} 10 \\
\mathrm{CR}(3) \mathrm{Cl} 10 \\
\mathrm{CR}(4) \mathrm{Cl} 10 \\
\mathrm{CR}(5) \mathrm{Cl} 10 \\
\mathrm{CR}(1) \mathrm{Cl} 11 \\
\mathrm{CR}(2) \mathrm{Cl} 11 \\
\mathrm{CR}(3) \mathrm{Cl} 11 \\
\mathrm{CR}(4) \mathrm{Cl} 11 \\
\mathrm{CR}(5) \mathrm{Cl} 11 \\
\operatorname{LP}(1) \mathrm{N} 9 \\
\operatorname{LP}(1) \mathrm{Cl} 10 \\
\operatorname{LP}(2) \mathrm{Cl} 10 \\
\operatorname{LP}(3) \mathrm{Cl} 10 \\
\operatorname{LP}(1) \mathrm{Cl} 11 \\
\operatorname{LP}(2) \mathrm{Cl} 11 \\
\operatorname{LP}(3) \mathrm{Cl} 11\end{array}$ & $\begin{array}{l}1.98950 \\
1.98955 \\
1.99372 \\
1.98893 \\
1.98975 \\
1.99964 \\
2.00000 \\
1.99979 \\
1.99992 \\
1.99998 \\
1.99999 \\
2.00000 \\
1.99979 \\
1.99995 \\
1.99995 \\
1.99999 \\
1.96370 \\
1.99350 \\
1.97008 \\
1.92962 \\
1.99348 \\
1.97021 \\
1.92814\end{array}$ & $\begin{array}{l}0.6793\left(\mathrm{sp}^{3.31}\right)_{\mathrm{C} 1}+0.7338\left(\mathrm{sp}^{4.77} \mathrm{~d}^{0.03}\right)_{\mathrm{Cl11}} \\
0.6794\left(\mathrm{sp}^{3.28} \mathrm{~d}^{0.01}\right)_{\mathrm{C} 2}+0.7338\left(\mathrm{sp}^{4.77} \mathrm{~d}^{0.03}\right)_{\mathrm{C} 10} \\
0.6400\left(\mathrm{sp}_{3.12} \mathrm{~d}^{0.01}\right)_{\mathrm{C} 8}+0.7684\left(\mathrm{sp}^{2.08}\right)_{\mathrm{N} 9} \\
0.8315\left(\mathrm{sp}_{3.14}\right)_{\mathrm{N} 9}+0.5555(\mathrm{~s})_{\mathrm{H} 19} \\
0.8297\left(\mathrm{sp}_{3.17}\right)_{\mathrm{N} 9}+0.5582(\mathrm{~s})_{\mathrm{H} 20} \\
\mathrm{~s} \\
\mathrm{~s} \\
\mathrm{~s} \\
\mathrm{p} \\
\mathrm{p} \\
\mathrm{p} \\
\mathrm{s} \\
\mathrm{s} \\
\mathrm{p} \\
\mathrm{p} \\
\mathrm{p} \\
\mathrm{sp}^{4.15} \\
\mathrm{sp}^{0.21} \\
\mathrm{sp}^{99.99} \mathrm{~d}^{0.11} \\
\mathrm{p} \\
\mathrm{sp}^{0.21} \\
\mathrm{sp}^{99.99} \mathrm{~d}^{0.11} \\
\mathrm{p}\end{array}$ \\
\hline
\end{tabular}

Table S6: Occupancy of natural orbitals (NBOs) and hybrids of chlorine derivatives calculated by the B3LYP method with 6-31G*(d) basis set

\begin{tabular}{|c|c|c|c|}
\hline Symbol & $\mathrm{NBO}^{\mathrm{a}}$ & Occupancy & Hybrid \\
\hline $\mathrm{Cl}(\mathrm{OH})-\mathrm{DOP}{ }^{*}$ & $\begin{array}{l}\sigma\left(\mathrm{C}_{1}-\mathrm{Cl}_{11}\right) \\
\sigma\left(\mathrm{C}_{2}-\mathrm{O}_{1} \mathrm{O}\right) \\
\sigma\left(\mathrm{C}_{8}-\mathrm{N}_{9}\right) \\
\sigma\left(\mathrm{N}_{9}-\mathrm{H}_{19}\right) \\
\sigma\left(\mathrm{N}_{9}-\mathrm{H}_{20}\right) \\
\sigma\left(\mathrm{o}_{10}-\mathrm{H}_{21}\right) \\
\mathrm{CR}(1) \mathrm{N} 9 \\
\mathrm{CR}(1) \mathrm{O} 10 \\
\mathrm{CR}(1) \mathrm{Cl} 11 \\
\mathrm{CR}(2) \mathrm{Cl} 11 \\
\mathrm{CR}(3) \mathrm{Cl} 11 \\
\mathrm{CR}(4) \mathrm{Cl} 11 \\
\mathrm{CR}(5) \mathrm{Cl} 11 \\
\operatorname{LP}(1) \mathrm{N} 9 \\
\operatorname{LP}(1) \mathrm{O} 10 \\
\operatorname{LP}(2) \mathrm{O} 10 \\
\operatorname{LP}(1) \mathrm{Cl} 11 \\
\operatorname{LP}(2) \mathrm{Cl} 11 \\
\operatorname{LP}(3) \mathrm{Cl} 11\end{array}$ & $\begin{array}{l}1.98873 \\
1.99426 \\
1.99377 \\
1.98909 \\
1.98975 \\
1.98795 \\
1.99964 \\
1.99980 \\
2.00000 \\
1.99979 \\
1.99994 \\
1.99996 \\
1.99999 \\
1.96402 \\
1.98244 \\
1.88683 \\
1.99388 \\
1.96992 \\
1.93318\end{array}$ & $\begin{array}{l}0.6777\left(\mathrm{sp}^{3.29} \mathrm{~d}^{0.01}\right)_{\mathrm{C} 1}+0.7353\left(\mathrm{sp}^{4.71} \mathrm{~d}^{0.03}\right)_{\mathrm{Cl} 11} \\
0.5792\left(\mathrm{sp}^{3.11} \mathrm{~d}^{0.01}\right)_{\mathrm{C} 2}+0.8152\left(\mathrm{sp}^{2.18}\right)_{\mathrm{O} 10} \\
0.6394\left(\mathrm{sp}^{3.13} \mathrm{~d}^{0.01}\right)_{\mathrm{C} 8}+0.7689\left(\mathrm{sp}^{2.07}\right)_{\mathrm{N} 9} \\
0.8311\left(\mathrm{sp}^{3.15}\right)_{\mathrm{N} 9}+0.5561(\mathrm{~s})_{\mathrm{H} 19} \\
0.8296\left(\mathrm{sp}^{3.17}\right)_{\mathrm{N} 9}+0.5584(\mathrm{~s})_{\mathrm{H} 20} \\
0.8645\left(\mathrm{sp}^{3.93} \mathrm{~d}^{0.01}\right)_{\mathrm{O} 10}+0.5026(\mathrm{~s})_{\mathrm{H} 21} \\
\mathrm{~s} \\
\mathrm{~s} \\
\mathrm{~s} \\
\mathrm{~s} \\
\mathrm{p} \\
\mathrm{p} \\
\mathrm{p} \\
\mathrm{sp}^{4.15} \\
\mathrm{sp}^{1.10} \\
\mathrm{sp}^{99.99} \mathrm{~d}^{0.18} \\
\mathrm{sp}^{0.21} \\
\mathrm{sp}^{99.99} \mathrm{~d}^{0.07} \\
\mathrm{p}\end{array}$ \\
\hline
\end{tabular}


NMR

Nuclear magnetic resonance (NMR) is very powerful tool in studying microscopic phenomena in physics, chemistry, biology and medicine. Determination of spectral parameters using the quantum-chemistry methods helps in the assignment and interpretation of the experimental data $^{32}$. In this study, NMR has been used for

Table S7: Occupancy of natural orbitals (NBOs) and hybrids of bromine derivatives

\begin{tabular}{|c|c|c|c|}
\hline Symbol & $\mathrm{NBO}^{\mathrm{a}}$ & Occupancy & Hybrid \\
\hline \multirow[t]{41}{*}{$(\mathrm{Br}) 2-\mathrm{DOP}{ }^{\wedge *}$} & $\sigma\left(\mathrm{C}_{1}-\mathrm{Br}_{11}\right)$ & 1.98553 & $0.7066\left(\mathrm{sp}^{3.52}\right)_{\mathrm{C} 1}+0.7076\left(\mathrm{sp}^{6.23} \mathrm{~d}^{0.02}\right)_{\mathrm{Br} 11}$ \\
\hline & $\sigma\left(\mathrm{C}_{2}-\mathrm{Br}_{10}\right)$ & 1.98570 & $0.7071\left(\mathrm{sp}^{3.48}\right)_{\mathrm{C} 2}+0.7071\left(\mathrm{sp}^{6.21} \mathrm{~d}^{0.02}\right)_{\mathrm{Br} 10}$ \\
\hline & $\sigma\left(\mathrm{C}_{8}-\mathrm{N}_{9}\right)$ & 1.99323 & $0.6383\left(\mathrm{sp}^{3.14}\right)_{\mathrm{C} 8}+0.7698\left(\mathrm{sp}^{2.19}\right)_{\mathrm{N} 9}$ \\
\hline & $\sigma\left(\mathrm{N}_{9}^{0}-\mathrm{H}_{19}\right)$ & 1.98916 & $0.8307\left(\mathrm{sp}^{3.21}\right)_{\mathrm{N} 9}+0.5567(\mathrm{~s})_{\mathrm{H} 19}$ \\
\hline & $\sigma\left(\mathrm{N}_{9}-\mathrm{H}_{20}\right)$ & 1.99077 & $0.8289\left(\mathrm{sp}^{3.26}\right)_{\mathrm{N} 9}+0.5594(\mathrm{~s})_{\mathrm{H} 20}$ \\
\hline & $\mathrm{CR}(1) \mathrm{N} 9$ & 1.99967 & $\mathrm{~s}$ \\
\hline & $\mathrm{CR}(1) \mathrm{Br} 10$ & 2.00000 & $\mathrm{~s}$ \\
\hline & $\mathrm{CR}(2) \mathrm{Br} 10$ & 1.99997 & $\mathrm{~s}$ \\
\hline & $\mathrm{CR}(3) \operatorname{Br} 10$ & 1.99976 & $\mathrm{~s}$ \\
\hline & $\mathrm{CR}(4) \mathrm{Br} 10$ & 1.99997 & $\mathrm{p}$ \\
\hline & $\mathrm{CR}(5) \mathrm{Br} 10$ & 1.99986 & $\mathrm{p}$ \\
\hline & $\mathrm{CR}(6) \mathrm{Br} 10$ & 1.99999 & $\mathrm{p}$ \\
\hline & $\mathrm{CR}(7) \operatorname{Br} 10$ & 1.99998 & $\mathrm{p}$ \\
\hline & $\mathrm{CR}(8) \mathrm{Br} 10$ & 2.00000 & $\mathrm{p}$ \\
\hline & $\mathrm{CR}(9) \mathrm{Br} 10$ & 1.99999 & $\mathrm{p}$ \\
\hline & $\mathrm{CR}(10) \mathrm{Br} 10$ & 1.99993 & $d$ \\
\hline & $\mathrm{CR}(11) \operatorname{Br} 10$ & 1.99993 & $d$ \\
\hline & $\mathrm{CR}(12) \mathrm{Br} 10$ & 1.99999 & $d$ \\
\hline & $\mathrm{CR}(13) \mathrm{Br} 10$ & 1.99980 & $d$ \\
\hline & $\mathrm{CR}(14) \mathrm{Br} 10$ & 1.99993 & $d$ \\
\hline & $\mathrm{CR}(1) \mathrm{Br} 11$ & 2 & $\mathrm{~s}$ \\
\hline & $\mathrm{CR}(2) \mathrm{Br} 11$ & 1.99997 & $\mathrm{~s}$ \\
\hline & $\mathrm{CR}(3) \mathrm{Br} 11$ & 1.99976 & $\mathrm{~s}$ \\
\hline & $\mathrm{CR}(4) \mathrm{Br} 11$ & 1.99998 & $\mathrm{P}$ \\
\hline & $\mathrm{CR}(5) \mathrm{Br} 11$ & 1.99994 & $\mathrm{P}$ \\
\hline & $\mathrm{CR}(6) \mathrm{Br} 11$ & 1.99998 & $\mathrm{P}$ \\
\hline & $\mathrm{CR}(7) \mathrm{Br} 11$ & 1.99991 & $\mathrm{P}$ \\
\hline & $\mathrm{CR}(8) \mathrm{Br} 11$ & 2 & $\mathrm{P}$ \\
\hline & $\mathrm{CR}(9) \mathrm{Br} 11$ & 1.99999 & $\mathrm{P}$ \\
\hline & $\mathrm{CR}(10) \mathrm{Br} 11$ & 1.99978 & $d$ \\
\hline & $\mathrm{CR}(11) \mathrm{Br} 11$ & 1.99998 & $d$ \\
\hline & $\mathrm{CR}(12) \mathrm{Br} 11$ & 1.99996 & $d$ \\
\hline & $\mathrm{CR}(13) \mathrm{Br} 11$ & 1.99994 & $d$ \\
\hline & $\mathrm{CR}(14) \mathrm{Br} 11$ & 1.99992 & $d$ \\
\hline & LP(1) N9 & 1.96207 & $\mathrm{sp}^{3.65}$ \\
\hline & LP(1) Br10 & 1.99455 & $\mathrm{sp}^{0.16}$ \\
\hline & LP(2) Br10 & 1.97317 & $\mathrm{sp}^{99.99} \mathrm{~d}^{0.13}$ \\
\hline & LP(3) Br10 & 1.93321 & $p$ \\
\hline & LP(1) Br11 & 1.99457 & $\mathrm{sp}^{0.16}$ \\
\hline & $\mathrm{LP}(2) \mathrm{Br} 11$ & 1.97342 & $s p^{99.99} d^{0.13}$ \\
\hline & LP(3) Br11 & 1.93271 & $p$ \\
\hline
\end{tabular}


determination of microscopic properties of dopamine and it halogenated derivate ${ }^{33-35}$.

To discuss the magnitude of the shielding tensor, it is necessary to report the three principal eigenvalues of the chemical shielding anisotropy tensor $\left(\sigma_{11}, \sigma_{22}, \sigma_{33}\right)$ using following equations:

a) the isotropic value of the shielding tensor which is defined as:

$$
\sigma_{\text {iso }}=\left(\sigma_{11}+\sigma_{22}+\sigma_{33}\right) / 3
$$

a) the anisotropy $(\Delta \sigma)$ of the tensor, given by:

$$
\Delta \sigma=\sigma_{33}-\left(\sigma_{11}+\sigma_{22}\right) / 2
$$

b) the shielding tensor asymmetry parameter ( $\eta$ given by:

$$
\eta=\frac{\left|\sigma_{22}-\sigma_{11}\right|}{\left|\sigma_{33}-\sigma_{i s o}\right|}
$$

The optimization and NMR calculation are done by density functional theory (DFT) using B3LYP method and the standard $6-31 G^{*}$ basis set in the gas phase. Table 2 and 3 presents the computed chemical shift for selected hydrogen, nitrogen and carbon atoms. According to this Table, anisotropic shielding value $(\Delta \sigma)$ for $\mathrm{C}_{8}$ and $\mathrm{N}$ atom of $\mathrm{F}(\mathrm{OH})$-Dopmolecule and $\mathrm{N}$ are negative values. Interestingly, among all of mentioned carbon atoms, $\mathrm{C}_{2}$ of $\mathrm{F}(\mathrm{OH})$-Dophas the lowest isotropic shielding value. The $\stackrel{\circ}{\eta} \eta$ and charges valuesare summarized in Table S1 and S2 ${ }^{36-38}$.

\section{NBO analysis}

NBO method gives useful information about interactions in both filled and virtual orbital

\begin{tabular}{|c|c|c|c|}
\hline Symbol & $\mathrm{NBO}^{\mathrm{a}}$ & Occupancy & Hybrid \\
\hline \multirow{28}{*}{$\mathrm{Br}(\mathrm{OH})-\mathrm{DOP}^{*}$} & $\sigma\left(\mathrm{C}_{1}-\mathrm{Br}_{11}\right)$ & 1.98444 & $0.7029\left(\mathrm{sp}^{3.53}\right)_{\mathrm{C} 1}+0.7113\left(\mathrm{sp}^{6.18} \mathrm{~d}^{0.02}\right)_{\mathrm{Br} 11}$ \\
\hline & $\sigma\left(\mathrm{C}_{2}-\mathrm{O}_{10}\right)$ & 1.99462 & $0.5776\left(\mathrm{sp}^{2.98} \mathrm{~d}^{0.01}\right)_{\mathrm{C} 2}+0.8163\left(\mathrm{sp}^{1.90}\right)_{\mathrm{O} 10}$ \\
\hline & $\sigma\left(\mathrm{C}_{8}-\mathrm{N}_{9}\right)$ & 1.9934 & $0.6375\left(\mathrm{sp}^{3.16} \mathrm{~d}^{0.01}\right)_{\mathrm{C} 8}+0.7705\left(\mathrm{sp}^{2.19}\right)_{\mathrm{N} 9}$ \\
\hline & $\sigma\left(\mathrm{N}_{9}^{0}-\mathrm{H}_{19}\right)$ & 1.9894 & $0.8302\left(\mathrm{sp}^{3.22}\right)_{\mathrm{N} 9}+0.5574(\mathrm{~s})_{\mathrm{H} 19}$ \\
\hline & $\sigma\left(\mathrm{N}_{9}-\mathrm{H}_{20}\right)$ & 1.99075 & $0.8286\left(\mathrm{sp}^{3.27}\right)_{\mathrm{N} 9}+0.5598(\mathrm{~s})_{\mathrm{H} 20}$ \\
\hline & $\sigma\left(\mathrm{o}_{10}-\mathrm{H}_{21}\right)$ & 1.98841 & $0.8642\left(\mathrm{sp}^{3.87} \mathrm{~d}^{0.01}\right)_{\mathrm{O} 10}+0.5031(\mathrm{~s})_{\mathrm{H} 21}$ \\
\hline & $\mathrm{CR}(1) \mathrm{N} 9$ & 1.99967 & $\mathrm{~S}$ \\
\hline & $\mathrm{CR}(1) \mathrm{O} 10$ & 1.99977 & $\mathrm{~s}$ \\
\hline & $\mathrm{CR}(1) \mathrm{Br} 11$ & 2 & $\mathrm{~s}$ \\
\hline & $\mathrm{CR}(2) \mathrm{Br} 11$ & 1.99997 & $\mathrm{~s}$ \\
\hline & $\mathrm{CR}(3) \mathrm{Br} 11$ & 1.99976 & $\mathrm{~s}$ \\
\hline & $\mathrm{CR}(4) \mathrm{Br} 11$ & 1.99998 & $\mathrm{p}$ \\
\hline & $\mathrm{CR}(5) \mathrm{Br} 11$ & 1.99992 & $\mathrm{p}$ \\
\hline & $\mathrm{CR}(6) \mathrm{Br} 11$ & 1.99998 & $\mathrm{p}$ \\
\hline & $\mathrm{CR}(7) \mathrm{Br} 11$ & 1.99993 & $p$ \\
\hline & $\mathrm{CR}(8) \mathrm{Br} 11$ & 2 & $p$ \\
\hline & $\mathrm{CR}(9) \mathrm{Br} 11$ & 1.99999 & $p$ \\
\hline & $\mathrm{CR}(10) \mathrm{Br} 11$ & 1.99978 & d \\
\hline & $\mathrm{CR}(11) \mathrm{Br} 11$ & 1.99996 & $d$ \\
\hline & $\mathrm{CR}(12) \mathrm{Br} 11$ & 1.99998 & $d$ \\
\hline & $\mathrm{CR}(13) \mathrm{Br} 11$ & 1.99994 & $d$ \\
\hline & $\mathrm{CR}(14) \mathrm{Br} 11$ & 1.99992 & $d$ \\
\hline & $\mathrm{LP}(1) \mathrm{N} 9$ & 1.96266 & $\mathrm{sp}^{3.63}$ \\
\hline & LP(1) 010 & 1.9786 & $\mathrm{sp}^{1.22}$ \\
\hline & LP(2) 010 & 1.86962 & $p$ \\
\hline & LP(1)Br11 & 1.9953 & $\mathrm{spp}^{0.16}$ \\
\hline & LP(2)Br11 & 1.97324 & $s p^{99.99} d^{0.02}$ \\
\hline & LP(3)Br11 & 1.93939 & $p$ \\
\hline
\end{tabular}
spaces which could enhance the analysis of intra

Table S8: Occupancy of natural orbitals (NBOs) and hybrids of bromine derivatives 
and intermolecular interactions. This is carried out by considering all possible interactions between filled donor and empty acceptor NBOs and estimating their energy importance by second order perturbation theory ${ }^{39}$.

NBO analysis is based on a method for optimally transforming a given wave function into localized form, corresponding to the one-center ("lone pairs") and two-center ("bonds") elements of the chemist's Lewis structure picture. In NBO analysis, the input atomic orbital basis set is transformed via natural atomic orbitals (NAOs) and natural hybrid orbitals (NHOs) into natural bond orbitals (NBOs) ${ }^{40-43}$.
Table 4-9 lists the calculated occupancies of natural orbitals. The calculated Natural bond hybrids and are also given in this table $e^{44-46}$.

Table S3-S8 show share of orbitals contribute in the bonds (BD for 2-center bond).

According to calculations, the nitrogen atom forms three single bond (sigma bond) with two hydrogen atoms $\mathrm{H}_{19}$ and $\mathrm{H}_{20}\left(\sigma_{\mathrm{N}_{9}}-\mathrm{H}_{19}\right.$ and $\sigma_{\mathrm{N}_{9}}$ $\left.\mathrm{H}_{20}\right)$, and a carbon atom $\mathrm{C}_{8}\left(\sigma_{\mathrm{C}_{9}}-\mathrm{N}_{9}\right)$ and a lon pair orbital (Lp). As seen from Table S3-S8, the LP on the nitrogen atom is formed from an $\mathrm{sp}^{3.62}$ hybrid for the $(\mathrm{OH})_{2}$-DOP molecule, $\mathrm{sp}^{4.15}$ hybrid for the $\mathrm{F}_{2}$-DOP molecule, $\mathrm{sp}^{3.63}$ For the $\mathrm{F}(\mathrm{OH})$-DOPmolecule, $\mathrm{sp}^{4.15}$ hybrid for the $\mathrm{Cl}_{2}$-DOP molecule, $\mathrm{sp}^{4.15}$ hybrid For

Scheme 1: Optimized structure of dopamine and halogenated derivatives

Fig. 1: The scan of potential energy verses degrees changing of Dopamine 
the $\mathrm{Cl}(\mathrm{OH})$-DOP molecule, $\mathrm{sp}^{3.65}$ hybrid for the $\mathrm{Br}_{2}$ DOP molecule, andsp ${ }^{3.63}$ hybrid For the $\mathrm{Br}(\mathrm{OH})$ DOP molecule ${ }^{47}$.

\section{CONCLUSION}

In the present work, atheoretical analysis of dopamine and its halogenated derivate has been performed in order to obtain the dipole moment $(\mu)$ and energy of structure formation (HF)of dopamine and it halogenated derivate. Also the thermodynamic parameters of this compound has been studied and according to the results, it has been found that the amount of Gibbs free energy $(\Delta G)$ and standard enthalpies $(\Delta H)$ of dopamine and its halogenated derivatearepositive value, therefore dopamine and its halogenated derivateareunstable structure. Moreover, the chemical shifts of these molecules have been simulated using quantum mechanics. Finally, the Natural Bond Orbital (NBO) analysis has provided the detailed insight into the type of hybridization and the nature of bonding in dopamine and its halogenated derivate.

\section{REFERENCES}

1. Dahlstrom, A.; Fuxe K. Experientia. 1964, 15, 398-399.

2. Tierney,L.; McPhee, S.;Papadakis, M. Quinta Edic. Edit. El Manual Moderno. 2000, 965967.

3. Abdel-Baki,A.;Ouetllet-Plamondon, C.; Malla,A. Journal of Affective Disorders. 2012, 138, S3.

4. Santos-García, D.;Prieto-Formoso,M.; de la Fuente-Fernández, R.Journal of Neurological Science. 2012, 318, 91.

5. Monajjemi,M.;Honarparvar, B. H.;Haeri, H.; Heshmat, M. Russian Journal of Physical Chemistry C. 2006, 80(1), S40-S44.

6. Mollamin,F.; Monajjemi, M.; Mehrzad J. Fullerenes, Nanotubes, and Carbon Nanostructures. 2014, 22: 738-751,

7. Monajjemi, M.;. Karachi, N.;.Mollaamin, F. Fullerenes, Nanotubes, and Carbon Nanostructures. 2014, 22, 643-662.

8. Tahan A.;Monajjemi, M. ActaBiotheor. 2011 , 59, 291-312.

9. Monajjemi, M.; Sobhanmanesh, A.; Mollaamin, F. Fullerenes, Nanotubes, and Carbon Nanostructures.2013, 21, 47-63.

10. Nicola, S.M.; Surmeier,J.;Malenka, R.C.Annu Rev Neurosci. 2000, 23, 185-215.

11. Monajjemi, M.; Robert Wayne, Jrand James E. Boggs, Chemical Physics. 2014,433, 1-11.

12. Ardalan, T.;Ardalan, P.;Monajjemi, M. Fullerenes, Nanotubes, and Carbon Nanostructures. 2014, 22, 687-708.

13. Monajjemi, M.; Mollaamin,F .J Clust Sci. 2012, 23, 259-272.
14. Monajjemi, M.; Chegini, H.; Mollaamin, F.; Farahani,Fullerenes P. Nanotubes, and Carbon Nanostructures. 2011, 19, 469-482.

15. Mollamin,F.; Monajjemi, M.; Mehrzad J. Fullerenes, Nanotubes, and Carbon Nanostructures. 2014, 22: 738-751.

16. Monajjemi, M.;Yamola, H.;Mollaamin, F. Fullerenes, Nanotubes, and Carbon Nanostructures. 2014, 22, 595-603.

17. Yahyaei, h.; Monajjemi, M. Fullerenes, Nanotubes, and Carbon Nanostructures. 2014, 22, 346-361.

18. Moleman, P.; van Berkum, H.; Tjaden,U.;de JongJ.Journal of Neuroscience Methods 11. 1984, 29.

19. Thierry,A.M.; Jay, T.M.; Pirot, S.; Mantz, J.; Godbout, R.; Glowinski, J. In: Motor and Cognitive Functions of the Prefrontal Cortex. (Thierry A.M., Glowinski J., Goldman- Rakic P.S., Christen Y., eds),.NewYork: SpringerVerlag.1994,pp 35-50.

20. Frisch, M.J.; Pople,J.A.;Binkley, J.S. J. Chem. Phys.1984, 80, 3265.

21. Gaussian 09, Revision A.02, M. J. Frisch et al. Gaussian, Inc., Wallingford CT, 2009.

22. Monajjemi, M.; Boggs, J.E. J. Phys. Chem A.2013, 117, 1670.

23. Mollaamin,F.; Monajjemi,M. J. Comput. Theor. Nanosci.2012, 9, 597-601.

24. Monajjemi, M.; Lee, V. S.; Khaleghian, M.; Honarparvar, B.; Mollaamin,F. J. Phys. Chem. C. $2010,114,15315-15330$.

25. Monajjemi, M. Chemical Physics. 2013, 425, 29-45. 
26. Yahyaei, H.; Monajjemi, M.; Aghaie, H., Zare K. Journal of Computational and Theoretical Nanoscience. 2013, 10(10), 2332-2341.

27. Monajjemi, M. Struct Chem.2012, 23, 551580.

28. Monajjemi, M.; JafariAzan M.; Mollaamin F.Fullerenes, Nanotubes, and Carbon Nanostructures.2013, 21(6) , 503-515.

29. Monajjemi, M.; Afsharnezhad, S.; Jaafari, M.R.; Abdolahi, T.; Nikosade, A.; Monajjemi, M. Russian Journal of physical chemistry A. 2007, 2, 1956-1963.

30. Mollaamin,F.; Monajjemi,M. Physics and Chemistry of Liquids.2012, 50(5), 596-604.

31. Monajjemi, M.; Faham R.; Mollaamin,F. Fullerenes, Nanotubes, and Carbon Nanostructures. 2012, 20, 163-169.

32. Vaara, J. CSC Report on Scientific Computing 1999-2000 .S. Kotila and J. Haataja, eds. CSC - Scientific Computing Ltd., Finland, 2001, 118-121.

33. Monajjemi, M.; Mahdavian,L.; Mollaamin,F.; Honarparvar,B.Fullerenes, Nanotubes and Carbon Nanostructures.2010, 18, 45-55.

34. Mollaamin, F.; Shahani pour, K.;Shahani pour, K.; Ilkhani, A. R.; Sheckari, Z.; Monajjemi M. 2012, 61(12), 2193-2198.

35. Monajjemi, M.; SeyedHosseini, M.; Mollaamin, F. Fullerenes, Nanotubes, and Carbon Nanostructures. 2013, 21, 381-393.

36. Monajjemi, M.; Falahati, M.; Mollaamin, F. Ionics. 2013, 19, 155-164.

37. Mollaamin, F.; Najafpour, J.; Ghadami,
S.;.Ilkhani, A. R.; Akrami, M. S.; Monajjemi, M.J. Comput. Theor.Nanosci.2014, 11, 12901298

38. Monajjemi, M.; Farahani N.; Mollaamin, F. Physics and Chemistry of Liquids.2012, 50(2), 161-172.

39. Reed, A.E.; Curtiss, L.A.; einhold,F.W.Chem Rev. 1988, 88 , 899.

40. Glendening,E. D.; Reed, A. E.;Carpenter, J. E.; Weinhold,F.Theoretical Chemistry Institute and Department of Chemistry, University of Wisconsin, Madison, Wisconsin 53706.

41. Monajjemi, M.;Mollaamin,F. J. Comput. Theor.Nanosci. 2012, 9, 2208-2214.

42. Mollaamin,F.; Baei,M.T.; Monajjemi, M.; Zhiani, R.; Honarparvar,B. Russian Journal of Physical Chemistry A. 2008, 82(13), 2354-2361.

43. Monajjemi, M.;Baheri, H.;Mollaamin,F. Journal of Structural Chemistry.2011, 52(1), 54-59.

44. Monajjemi, M.;Mahdavian,L.; Mollaamin, F. Bull Chem. Soc. Ethiop. 2008, 22(2), 1-10.

45. Mollaamin,F.; Varmaghani, Z.;Monajjemi, M.Physics and Chemistry of Liquids. 2011, 49(3), 318-336.

46. Monajjemi, M.;Mollaamin,F.;Karimkeshteh, T.J. Mex. Chem. Soc. 2005, 49(4), 336-340.

47. Monajjemi, M.;Najafpour, J.; Mollaamin, F. Fullerenes, Nanotubes, and Carbon Nanostructures.2013, 21, 213-232. 